\title{
Undergraduate Dermatology Curriculum: Perspectives of General Practitioners from Western India
}

\author{
Thakkar, S. H. ${ }^{1}$, Chavda, P. D. ${ }^{2}$, Mehta, K. G. ${ }^{2}$ \& Shringarpure, K. $^{3}$
}

\begin{abstract}
Introduction: Curriculum revision starts with gathering perspectives of different stakeholders including practicing doctors. It is important to take especially General Practitioners' (GPs) perspective while revising dermatology curriculum.
\end{abstract}

Methods: This cross-sectional needs assessment study was done among 61 purposively selected allopathic GPs working in rural and urban areas of a district located in western India. We used a validated semi-structured self-administered questionnaire. Quantitative findings are presented using proportions and narrative responses were analyzed by qualitative content analysis.

Results: The GPs recommended using a combination of theory lectures and clinical postings for dermatology teaching. 36 (59\%) participants suggested multiple clinical postings, 52 (85\%) recommended a four to eight weeks clinical posting and all suggested to make the internship posting mandatory in dermatology. They recommended outdoor patient exposure (93\%), case demonstration $(56 \%)$, and case presentation (51\%) as educational methods. $43(70 \%)$ and $32(52 \%)$ participants recommended Image Based Assessment and spot cases respectively as preferred method of assessment. $40(66 \%)$ practitioners suggested $10-20 \%$ weight-age of dermatology in summative assessment. They recommended that the syllabus should give more weight-age to the common dermatological problems and cutaneous adverse drug reactions.

Discussion: The practitioners suggested that the duration of the dermatology teaching should be increased looking at the quantum of the skin patients in general practitioner's outdoor patient department (OPD). They suggested an adequately long duration for posting in which a complete follow up care can be demonstrated to students on same patient. They recommended use of visual tools for teaching and assessment in dermatology.

Keywords: Needs Assessment, Skin, MBBS, Medical Education, Indian Medical Graduate, Dermatology Posting, Curriculum Planning

\section{Introduction}

An Indian medical graduate should be able to manage common skin diseases and understand need for referral services for the same as per the Medical Council of India regulations (Medical Council of India, 1997).

\footnotetext{
${ }^{1}$ Department of Skin \& VD, GMERS Medical College, Gotri, Vadodara, India.

${ }^{2}$ Department of Community Medicine, GMERS Medical College, Gotri, Vadodara, India.

${ }^{3}$ Department of Preventive and Social Medicine, Medical College, Vadodara, India.

Corresponding Author: Dr. Paragkumar Chavda Department of Community Medicine, GMERS Medical College, Gotri, Vadodara, India.

Email: paragchavda@gmail.com
}

DOI: http://doi.org/10.4038/seajme.v14i2.274
However, published research reports have suggested that graduating medical students and practicing GPs are not adequately competent compared to what is expected from them (Kaliyadan, 2010; Patro et al., 2015). One of the possible reasons for this may lie in dermatology curriculum in practice at medical schools (Kaur \& Singh, 1995). This calls for a need to revisit the undergraduate dermatology curriculum.

Curriculum planning is a formal process which starts with the general needs assessment as its first step; which should aim to take perspectives of the patients, health professionals, educators, and the community (Kern, 2009). Few studies are available from various countries suggesting need for curriculum change by surveying 
different population like medical students, dermatology teachers, educational leaders and GPs (Chiang et al., 2011; Ulman et al., 2015; Gupta et al., 2017; Malik et al., 2017). Kaliyadan has reported medical teachers' opinion on dermatology curriculum in India (Kaliyadan, 2010). The existing Indian literature is lacking in the practicing doctor's perspective on the dermatology curriculum.

Patients with skin problems commonly presents to GPs, dermatologists, pediatricians and internal medicine experts. The GPs are first point of contact for majority of the population for any ailment including skin problems. Skin disorders are the fourth most common cause of consultation to GPs (Salvi et al., 2015). Considering this need, we undertook this study to collect the general physician's perspective on undergraduate dermatology curriculum.

\section{Methods}

Ethical issues: Institutional Ethics Committee approval was then before starting the study.

Study design \& period: This was a cross sectional study. The data collection was done from May 2016 to July 2016.

Study setting \& participants: This study was done in a district located in western India. The team of researchers for this study was faculty from a medical college located in the same district.

Allopathic GPs working in government set up in this district were taken in the sampling frame of eligible participants. There were a few allopathic doctors working on administrative posts in government set up. Since their work profile involved largely the administrative work, they were excluded. We had a list of 52 and 34 GPs in the sampling frame of our study from the rural and urban area of a district respectively. Since this was a cross-sectional study only those practitioners available during single point of contact were included in the study. A total of 34 and 27 physicians were available from rural and urban area respectively during this point of contact for the data collection. Hence, the total sample consisted of 61 GPs. We used purposive sampling to include only GPs from a district for this study.

\section{Study instrument}

We prepared a questionnaire to gather GPs' perspective on undergraduate dermatology curriculum. The study instrument was validated by two faculty members from dermatology and two members of medical education unit of the institute. This version was pilot tested on a group of 5 medical officers from another district before implementing it on the actual study participants.

The study instrument was a semi structured self-administered questionnaire. The questionnaire collected background details of physicians, quality of exposure they received in dermatology during undergraduate course, their suggestions regarding exposure in dermatology during undergraduate course including internship, teaching-learning method, and assessment. An open-ended question at the end of the questionnaire collected their other suggestions for dermatology curriculum. The questionnaire was prepared and administered in English language.

A part of the questionnaire was focused on their training need assessment for common dermatological disorders using computer assisted image-based assessment (IBA), which is not presented here.

\section{Data collection}

Monthly review meetings are routinely conducted with GPs working as medical officers by both the rural and urban health authorities. We utilized the opportunity of the monthly review meeting at both urban and rural areas to get access to the GPs for collecting the data. Prior permission from the Chief District Health Officer for rural area and Chief Medical Officer for urban area were taken. Informed consent was taken from the study participants before collecting the data.

\section{Data analysis}

The quantitative data were entered and analyzed in Microsoft office excel spreadsheet 2013. The data were presented using proportion and averages with standard deviation. Qualitative data was analyzed by manual content analysis (Saldana, 2010). First author (dermatologist) and second author (research expert) separately analyzed the qualitative responses to open ended questions. Inductive approach was used to identify the emerging themes during this analysis. The discrepancies were reviewed by third author (research expert) and resolved through discussion. The themes and message emerging from this analysis is presented in tabular and narrative form in this paper. 


\section{Results}

We could collect responses from 61 GPs out of the total 76 GPs working in rural and urban area of the single district.

\section{Background information of participating GPs}

Among the participating GPs 34 (56\%) were from rural area and rest from urban area. The male: female ratio was 1.5:1 among them. Almost $40 \%$ physicians were under 30 years of age. Nearly half of the participants had less than 10 years of experience.

42 (69\%) GPs received their undergraduate medical education from Gujarat, rest of them received it from other parts of India (Table 1). $37(60 \%)$ physicians had received two weeks of dermatology posting during their own MBBS study. The practitioners reported that patients presenting with skin problems formed around $17 \%$ of the total out-patient attendees in their clinics. One third of the physicians frequently felt the need for referring skin patients to a dermatologist while the rest felt this sometimes.

Table 1: Demographic details of participating General Practitioners (n=61)

\begin{tabular}{lc}
\hline \multicolumn{1}{c}{ Demographic Variables } & $\mathbf{n}(\%)$ or mean \pm SD \\
\hline $\begin{array}{l}\text { Location } \\
\text { Rural } \\
\text { Urban }\end{array}$ & $34(56)$ \\
Gender & $27(44)$ \\
$\quad$ Male & \\
$\quad$ Female & $37(61)$ \\
Age (years) & $24(39)$ \\
Clinical practice experience (years) & $36.1 \pm 9.4$ \\
Place of MBBS study & $9.7 \pm 9.2$ \\
$\quad$ Gujarat & \\
States other than Gujarat & $42(69)$ \\
\end{tabular}

\section{Practitioners' recommendations on curriculum}

We asked the practitioners their recommendations on the organization of dermatology teaching, the teaching learning methods and assessment pattern.

58 (95\%) physicians recommended using a combination of theory lectures along with clinical posting rather than having any one method used alone. Only 2 (3\%) physicians opined that only clinical posting without theory lectures can suffice. None of them were in favor of teaching through only theory lectures. 36 $(60 \%)$ physicians recommended teaching dermatology over multiple clinical postings compared to one posting. $36(60 \%)$ participants recommended to keep the posting during third professional year while $30 \%$ recommended the postings to be scattered across all three clinical years.

Around 4-8 weeks long dermatology posting was suggested by 52 (85\%) of the participants. With regard to exposure to dermatology during internship, 38 (62\%) GPs recommended 4 weeks compulsory posting during internship (Table 2).

The participants suggested various teachinglearning methods for dermatology, either alone or in combination. The preferred method of learning suggested by them in decreasing order of frequency were outdoor patient exposure 57 (93\%), case demonstration 34 (56\%), case presentation $31(51 \%)$, atlas or computer assisted learning 16 (26\%), lecture 14 (23\%) and online learning modules 5 (8\%) (Figure 1). 


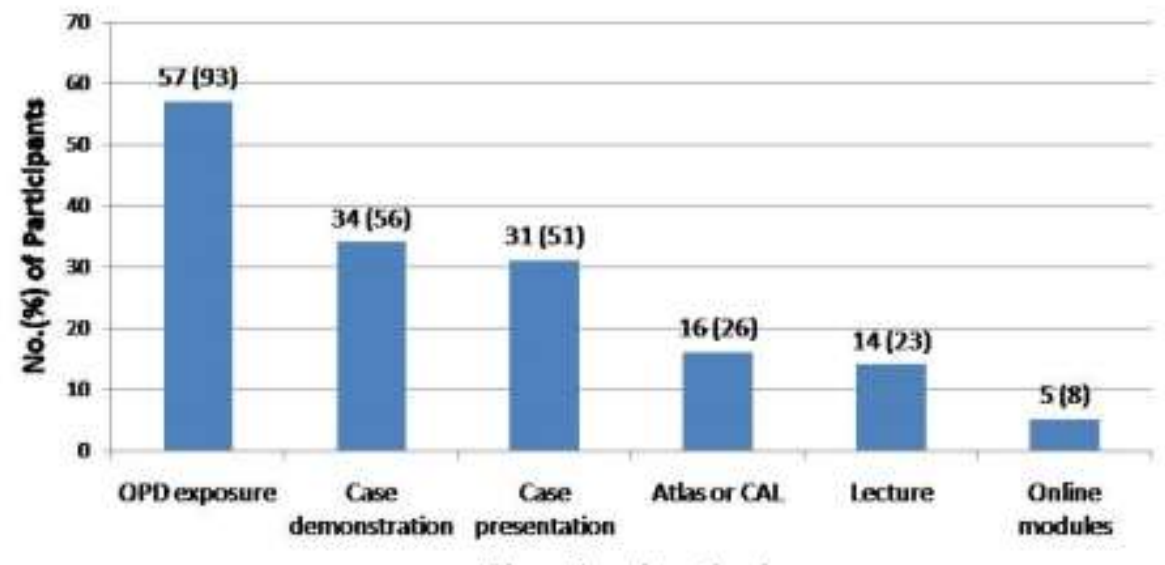

Educational methods

Figure 1: Suggestions of General Practitioners on preferred educational methods to be used in dermatology during MBBS (n=61)

CAL: Computer Assisted Learning

The physicians recommended various methods for assessment of students' learning in dermatology. Majority of the participants had recommended IBA $(70 \%)$ followed by spot cases $(52 \%)$, viva $(23 \%)$, and theory exam (21\%). $10 \%$ physicians also recommended to use objective structured clinical examination (OSCE). The physicians were also asked their opinion on the weight-age of marks from dermatology in summative assessment. None of the physician recommended a zero percent weight-age. $40 \quad(71 \%)$ participants recommended to have the dermatology weightage to be in range of $10-20 \%$ of total marks (Table 3).

Table 2: Recommendations by General Practitioners regarding dermatology posting during MBBS $(n=61)$

\begin{tabular}{lc}
\multicolumn{1}{c}{ Recommendations } & $\mathbf{n}(\%)$ \\
\hline Number of dermatology postings & $25(41)$ \\
Single & $36(59)$ \\
Multiple & \\
Appropriate time of posting & $6(10)$ \\
Second year & $12(20)$ \\
Third year Part I & $24(39)$ \\
Third year part II & $19(31)$ \\
Scattered in all three years & \\
Duration of posting during MBBS (weeks) & $3(5)$ \\
Two & $52(85)$ \\
Four- Eight & $6(10)$ \\
Twelve & \\
Duration of posting during internship (weeks) & $6(10)$ \\
Two & $38(62)$ \\
Four & $17(28)$ \\
Six- Eight & \\
\hline
\end{tabular}


Table 3: Recommendations of General Practitioners regarding assessment of dermatology learning during MBBS

\begin{tabular}{lc}
\hline \multicolumn{1}{c}{ Recommendations } & $\mathbf{n}(\%)$ \\
\hline Recommended method of assessment (n=61) & \\
Image Based Assessment & $43(70)$ \\
Spot cases & $32(52)$ \\
Viva & $14(23)$ \\
Theory exam & $13(21)$ \\
Objective structured clinical examination & $6(10)$ \\
Recommendation of weight-age of dermatology during & \\
summative assessment (n=58) & \\
$0 \%$ & 0 \\
$5 \%$ & $11(18)$ \\
$10 \%$ & $16(26)$ \\
$20 \%$ & $24(39)$ \\
$30 \%$ & $7(11)$ \\
\hline
\end{tabular}

An open-ended question inviting their suggestions on dermatology curriculum was responded by 36 (59\%) participants. Total 65 suggestions were received. The main suggestions were divided into four broad themes as syllabus, organization of dermatology teaching, teaching learning methods and assessment methods. Although many suggestions were a repetition of their responses on structured questions, we also received novel information as responses to this open-ended question (Table 4).
With regard to the organization, participants mentioned to emphasize the importance of skin problem in curriculum. This was also reflected in their suggestions for having a regular and multiple clinical posting and compulsory internship posting in dermatology. Two participants suggested to make available to the students the standard treatment guidelines in dermatology. One participant suggested that dermatology teaching should be done by professors rather than resident doctors.

Table 4: List of suggestions by General Practitioners on curriculum prepared using content analysis of open-ended responses (total 65 suggestions)

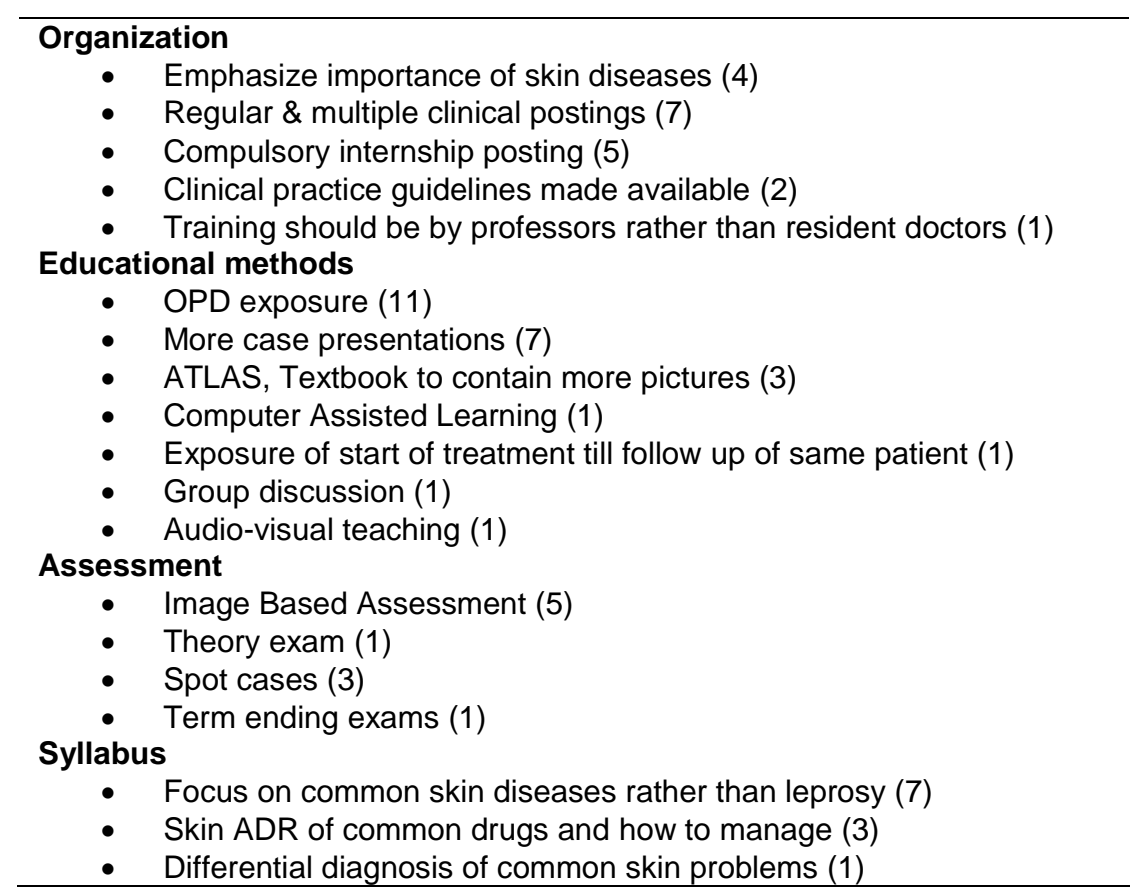


About the teaching learning method, many suggestions were pertaining to OPD exposure of students and case presentations by students. There were suggestions on using colored atlas, photographs, and textbook to contain more pictures. One participant mentioned that student should be exposed to the patient from start of treatment to follow up care of the same patient to understand the continuum of treatment.

The suggestions of using IBA, theory exam and spot cases were repetitions of their responses on structured questions. One participant particularly mentioned to ensure taking a term ending exam in dermatology.

The participants mentioned that in dermatology syllabus, the focus should be on common skin problems presenting in clinic rather than only on leprosy or HIV infections. Three participants also mentioned to include in curriculum the adverse reactions of commonly used drugs manifesting on skin and the management of such reactions. One participant mentioned to ensure teaching of how to arrive at a differential diagnosis of common skin presentations.

\section{Discussion}

What this study adds to the existing literature on recommendations for dermatology curriculum is the GPs' perspective.

\section{Summary of results}

The GPs opined that the dermatology was underrepresented in undergraduate curriculum. They suggested that the importance of common skin problems should be adequately emphasized in the curriculum. They suggested increasing the duration of skin posting during undergraduate course as well as internship. They also suggested to include more case exposure in the teaching methodology and use of more visuals in both teaching as well as assessment. They also recommended having weight-age of dermatology in university exams.

\section{Interpretation and implications}

The recommendations by GPs should be viewed in line with the fact that as high as $20 \%$ of their outpatient attendees present with dermatological problems. Nearly one third GPs feeling the need to frequently refer skin patients to the dermatologist indicates the need for larger representation of dermatology in undergraduate curriculum. Kerr et al also concludes that the gap in GPs' knowledge for dermatological problems underscores the importance of dermatology exposure in undergraduate teaching (Kerr et al., 2006).

\section{Organization of exposure in dermatology}

Nearly $60 \%$ of our participants received only 2 weeks clinical posting. The medical council of India guideline suggests clinical posting of 6 weeks (out of 142 weeks) duration along with 30 hours (out of 1440 hours for clinical subjects) of didactic teaching in dermatology; thus the allocation to dermatology comprising $2-4 \%$ of total undergraduate teaching time (Medical Council of India, 1997). In practice, many of the institutes have the schedule of clinical exposure of dermatology only for 2-4 weeks. The suggestions by our participants to spread the clinical posting across multiple years are also in agreement with the $\mathrm{MCl}$ guidelines and suggestions by other educators (Medical Council of India, 1997; Salvi et al., 2015). As per $\mathrm{MCl}$ guideline, the 2-week clerkship in dermatology during internship, is elective in nature (Medical Council of India, 1997). A student gets a list of 5 subjects to choose from apart from dermatology. Thus, all the GPs may not have had an opportunity for such clerkship. Those who would have opted for would have received a 2 weeks clerkship. More than half of our participants recommended a compulsory 4 weeks internship posting in dermatology. $\mathrm{MCl}$ has suggested three postings of two weeks for dermatology over a span of three \& half year of clinical exposure during MBBS. Looking to the curriculum we opine that; it is preferable to have eight weeks posting divided in two parts of four weeks each as two weeks is too short period to observe the follow up and treatment response in patients. We also believe that students must have four weeks mandatory internship posting in dermatology.

The literature suggests that efforts have been made at mainstreaming dermatology component of undergraduate curriculum in many countries. After several reviews the Canadian dermatology curriculum for undergraduate now contains more hours, increased number of teachers engaged and alignment of syllabus to the real-world priorities (Hu \& Vender, 2018). A paper from Australia reports the underrepresentation of dermatology in undergraduate curriculum and need for reforms (Gupta et al., 2017). The American Academy of Dermatology has produced an online curriculum for dermatology looking at the limitations and variability in dermatology education in USA (Cipriano et al., 2013). Though the British Association of Dermatology 
recommendations on undergraduate dermatology curriculum were published in 2006 recent papers still show the need for streamlining the dermatology component in undergraduate curriculum (Chiang et al., 2011; Shah et al., 2015).

The participants also recommended to make available the standard treatment guidelines for common skin problems to the students. Such standard treatment guidelines are prepared by some states in India now (Sundararaman et al., 2003; Desai et al., 2013). If such standard treatment guidelines are recognized as part of curriculum it may save time of the students in learning treatment approach for dermatology.

The participant from our study recommending that dermatology be taught by professors rather than resident doctors might have stemmed from their observation of resident doctors teaching a large portion of dermatology to undergraduate students. Resident doctors might not be adequately prepared for the teaching job. Hence, this suggestion is worth consideration by the teaching dermatology departments.

\section{Teaching-learning methods}

The GPs emphasized the OPD exposure and case presentation / demonstration as preferred methods of learning in dermatology. Dermatology, being a visual branch, clinical exposure can improve the ability and confidence in managing common skin disorders for GPs (Ulman et al., 2015). A survey among final year medical students from UK also reported clinical exposure and small group learning as an influential modality in increasing confidence level in dermatology (Chiang et al., 2011).

E-learning was suggested by one of the participants in our study. E learning or computer assisted learning becomes particularly relevant in our scenario of limited clinical hours of dermatology exposure (Silva et al., 2011). Another approach is use of more visuals. Our participants have suggested to make available colored atlas of common skin problems and the dermatology textbooks to contain more pictures. They also suggested to use more pictures and visuals in teaching as well as assessment. Kumar et al has documented that image-based teaching can serve as a very good supplement to routine clinical exposure (Kumar et al., 2015).

An interesting suggestion which came from the participants in this study was that the students should be exposed to the same patient from start of treatment till complete follow up. Our verbal communication with the GPs revealed that many GPs face difficulty dealing with patients during follow up or among patients with chronic skin problems. In the current practice of short 2 weeks clinical posting in dermatology it becomes challenging to teach follow up care to the students on the same case. This point also underscores the need for a longer duration of dermatology posting as suggested by them not only during the clinical postings but also during internship phase. Introduction of new element of Competency Based Curriculum in form of Clinical clerkship can be helpful to address this issue if the students are posted for a longer duration in dermatology at a stretch (Medical Council of India, 2019).

\section{Assessment}

Considering GPs' load for the patients with common skin disorders, it is important to ensure clinical competency of graduating medical students to deal with common skin disorders. As assessment drives learning, the role of assessment in dermatology curriculum is important.

Nearly three fourth of the participants recommended having $10-20 \%$ of weight-age of dermatology in summative assessment. The current practice at many institutes is to include dermatology in only the theory exam at summative assessment; the weight-age is also very small at around $2-3 \%$ of total marks. The participants suggested to ensure the conduct of term ending examinations in clinical postings. Currently, Formative assessment is being conducted in form of viva or theory exams at many institutes. Competency Based curriculum has made it compulsory to attain $50 \%$ score in each term ending exam to become eligible for the university exam is a welcome step looking to the suggestion of the participants (Medical Council of India, 2019). The practitioners suggested to have assessment with visual mode using spot case or IBA to have constructive alignment with objectives.

In our sample of GPs, we observed that the most frequently suggested method of assessment was IBA. Higher proportion for the choice of IBA may be due to their recent exposure to IBA as a part of training needs assessment. Very few participants in this study suggested to use OSCE as probably, they might not be aware of the concept of OSCE. 
Few of the dermatology departments have tried computer assisted OSCE and found it effective in assessing competency in dermatology (Grover et al., 2012; Kaliyadan et al., 2014; Chaudhary et al., 2017).

\section{Syllabus}

The practitioners suggested to focus on the common dermatological problems presenting to OPD. They observed that large focus is given to leprosy/HIV in undergraduate curriculum but the other common skin problems should also be given adequate weight-age. The participants also suggested to include the cutaneous adverse reactions of commonly used drugs and their management in the syllabus. The physicians mentioned difficulty in arriving at correct diagnosis for skin patients. Hence, they suggested to use a symptom based approach of differential diagnosis of commonly presenting skin lesions in the syllabus. The current dermatology syllabus as it is taught and presented in books follows a disease-oriented approach. Hence the physicians favored to use a more physician friendly organization of the syllabus. A paper on Malaysian medical undergraduate dermatology curriculum also underscores the need for prioritizing locally prevalent skin conditions in the curriculum (Jamil et al., 2016).

\section{Strengths and Limitations}

The strength of this study is we covered a relatively large sample of GPs. Our sample of GPs was representative. Physicians practicing in rural and urban area; the senior and junior physicians and physicians who had their undergraduate education from Gujarat as well as outside Gujarat were represented in our study sample.

Our quantitative survey findings were supplemented with robust qualitative analysis of the open-ended responses by the participants. Our team members trained in qualitative researchers enabled us to carry out this analysis.

The limitation of the study was that we could reach out to only those practitioners working in government set up. The GPs working in private sector were not included in our study. The syllabus part of the curriculum was covered through qualitative approach only in this study.

\section{Future directions}

Curriculum revision should be considered looking to the burden of common skin disorders. Future studies gathering perspectives of GPs from private set up as well as GPs from other parts of the country are worth exploring. This study focused only on general practitioner's perspective. It is important to explore perspectives of other stake holders like practicing doctors of other specialties, dermatology teachers, and society in general as part of the curriculum needs assessment.

\section{Conclusion}

Dermatology curriculum needs to be aligned with community needs as discovered from the GPs' perspective in this study. GPs recommended increased exposure in dermatology along with compulsory internship posting in dermatology. They recommended to prioritize the common skin problems presenting at OPD in dermatology syllabus. They recommended use of visual tools for teaching and assessment and an increased weight-age of dermatology in summative assessment.

\section{Acknowledgements}

We would like to thank Advance Course in Medical Education (ACME) faculties and colleagues for their guidance and help as it was a part of ACME project. A special thanks to Dr. $P$. V. Vijayaraghavan, for his valuable help in conceptualization of this study.

This manuscript also benefitted from the faculty training program in health research conducted by the central research unit of GMERS Medical College, Vadodara. We thank the faculty members who served as mentors and peers during this training program and contributed to improving the quality of this research study. We thank the Dean and Medical Superintendent for supporting this research project.

We would like to thank Dr. Chhari, Chief District Health Officer, Vadodara and Dr. Devesh Patel, Medical Officer of Health, Vadodara Municipal Corporation for providing access to the PCP for conducting this study during their scheduled meetings. We thank Dr. Raksha Patel and Dr. Roshni Vahora from the Department of Dermatology, at GMERS Medical College, Vadodara for their help. 


\section{References}

Chaudhary, R., Grover, C., Bhattacharya, S.N. \& Sharma, A. (2017). Computer Assisted Objective Structured Clinical Examination versus Objective Structured Clinical Examination in Assessment of Dermatology Undergraduate Students. Indian Journal of Dermatology, Venereology, and Leprology, 83, 4, p. 448.

Chiang, Y.Z., Tan, K.T., Chiang, Y.N., Burge, S.M., Griffiths, C.E. \& Verbov, J.L. (2011). Evaluation of Educational Methods in Dermatology and Confidence Levels: A National Survey of UK Medical Students. International Journal of Dermatology, 50, 2, pp. 198-202.

Cipriano, S.D., Dybbro, E., Boscardin, C.K., Shinkai, K. \& Berger, T.G. (2013). Online Learning in a Dermatology Clerkship: Piloting the New American Academy of Dermatology Medical Student Core Curriculum. Journal of the American Academy of Dermatology, 69, 2, pp. 267-272.

Desai, M., Shah, S. \& lyer, G. (eds) (2013). Standard Treatment Guidelines: A Manual for Medical Therapeutics. 1st edn. Gandhinagar: Gujarat Medical Services Corporation Limited, Health \& Family Welfare Department, Government of Gujarat.

Grover, C., Bhattacharya, S.N., Pandhi, D., Singal, A. \& Kumar, P. (2012). Computer Assisted Objective Structured Clinical Examination: A useful Tool for Dermatology Undergraduate Assessment. Indian Journal of Dermatology, Venereology, and Leprology, 78, 4, p. 519.

Gupta, A., Chong, A., Scarff, C. \& Huilgol, S. (2016). Dermatology Teaching in Australian Medical Schools. Australasian Journal of Dermatology, 57.

$\mathrm{Hu}$, A. \& Vender, R., 2018. Undergraduate Dermatology Education in Canada: A National Survey. Journal of Cutaneous Medicine and Surgery, 22, 1, pp. 31-37.

Jamil, A., Muthupalaniappen, L., Nor, N.M., Siraj, H.H. \& Salam, A. (2016). Identifying the Core Content of a Dermatology Module for Malaysian Medical Undergraduate Curriculum Using a Modified Delphi Method. The Malaysian Journal of Medical Sciences: MJMS, 23, 3, p. 78.

Kaliyadan, F. (2010). Undergraduate Dermatology Teaching In India: Need for Change. Indian Journal of Dermatology, Venereology, and Leprology, 76, 5, p. 455.

Kaliyadan, F., Khan, A.S., Kuruvilla, J. \& Feroze, K. (2014). Validation of a Computer Based Objective Structured Clinical Examination in the Assessment of Undergraduate Dermatology Courses. Indian Journal of Dermatology, Venereology, and Leprology, 80, 2, p. 134.
Kaur, P. \& Singh, G. (1995). Community Dermatology in India. International Journal of Dermatology, 34, 5, pp. 322-322.

Kern, D.E. (2016). A Six-Step Approach to Curriculum Development. $P$ Thomas, D Kern, M, Hughes, \& B Chen, Curriculum Development for Medical Education, pp. 5-9.

Kerr, O.A., Walker, J. \& Boohan, M. (2006). General Practitioners' Opinions Regarding the Need for Training in Dermatology at Undergraduate and Postgraduate Levels. Clinical and Experimental Dermatology, 31, 1, pp. 132-133.

Kumar, G.R., Madhavi, S., Karthikeyan, K. \& Thirunavakarasu, M.R. (2015). Role of Clinical Images Based Teaching as a Supplement to Conventional Clinical Teaching in Dermatology. Indian Journal of Dermatology, 60, 6, p. 556.

Malik, M. H., Khizrah, A., Ghulam, M., Raheel, T., Kashif, I., Sumera, Y., Madiha, A., Saman, Z., Saira, S., Asma, N., Fatima, A., Nazia, H., Samreen, K., Zara, Z., Sadaf, S., Aroosha, Z. \& Maryam, M. (2017). 'Medical School Dermatology Curriculum - Are Our Graduates Adequately Prepared and Aligned to Community Needs?', Journal of Sheikh Zayed Medical College, 8, 2, pp. 1147-1152.

Medical Council of India (1997). Salient Features of Regulations on Graduate Medical Education, 1997. Published in Part iii, Section 4 of the Gazette of India Dated 17th May 1997. Available at: https://old.mciindia.org/Rules-andRegulation/GME_REGULATIONS.pdf [Accessed: 5/9/2018].

Medical Council of India (2019). Regulations on Graduate Medical Education (Amendment), 2019.

Patro, B.K., Tripathy, J.P., De, D., Sinha, S., Singh, A. \& Kanwar, A.J. (2015). Diagnostic Agreement between a Primary Care Physician and a Teledermatologist for Common Dermatological Conditions in North India. Indian Dermatology Online Journal, 6, 1, p. 21.

Saldaña, J. (2015). The Coding Manual for Qualitative Researchers. Sage.

Salvi, S., Apte, K., Madas, S., Barne, M., Chhowala, S., Sethi, T., Aggarwal, K., Agrawal, A. \& Gogtay, J. (2015). Symptoms and Medical Conditions in 204912 Patients Visiting Primary Health-Care Practitioners in India: A 1-Day Point Prevalence Study (The POSEIDON Study). The Lancet Global Health, 3, 12, pp. e776-e784.

Shah, H., Pozo-Garcia, L. \& Koulouroudias, M., (2015). Dermatology-A Compulsory Part of the UK Medical School Curriculum? Medical Education Online, 20, 1, p. 30212. 
Silva, C. S., Souza, M. B., Filho, R. S. S., de Medeiros, L. M. \& Criado, P. R. (2011). 'ELearning Program for Medical Students in Dermatology', Clinics. Hospital das Clinicas da Faculdade de Medicina da Universidade de Sao Paulo, 66, 4, pp. 619-622.

Sundararaman, T., Singh, P. D. \& Dabke, A. (eds) (2003). Standard Treatment Guidelines for Medical Officers. Raipur: Department of Health \& Family Welfare, Government of Chhattisgarh.
Ulman, C.A., Binder, S.B. \& Borges, N.J. (2015). Assessment Of Medical Students' Proficiency in Dermatology: Are Medical Students Adequately Prepared to Diagnose and Treat Common Dermatologic Conditions in the United States? Journal of Educational Evaluation for Health Professions, 12, p. 18. 